\title{
La co-creación como estrategia para abordar la gobernanza de TI en una organización
}

\author{
Mauricio González ${ }^{1}$, Liliana González ${ }^{2}$ \\ magonzalez@udem.edu.co, ligonzalez@udem.edu.co \\ ${ }^{1}$ Profesor tiempo completo, Facultad de Ingeniería Universidad de Medellín-Medellín, Colombia. \\ ${ }^{2}$ Profesora tiempo completo, Facultad de Ingeniería Universidad de Medellín-Medellín, Colombia.
}

DOI: 10.17013/risti.15.1-16

Resumen: Dentro de los marcos comunes para la Gobernanza de Tecnologías de Información -TI- un principio fundamental es la identificación y satisfacción de las necesidades de los interesados dentro y fuera de las organizaciones. Sin embargo, las propuestas existentes no documentan procedimientos que apoyen dicha ejecución. La co-creación es una estrategia interesante para capturar tales necesidades, generando una visión compartida de las unidades de una compañía. Este artículo propone un método basado en co-creación para ejecutar la fase de identificación de necesidades en un proceso de gobernanza TI. Se detallan cinco componentes que permiten determinar estrategias, tipos de perfiles de los interesados, recursos requeridos durante la puesta en marcha y mecanismos de motivación. Al emplear el método, se evidenció un aumento significativo en el flujo de aportes, así como la creación de estrategias innovadoras para satisfacer las necesidades dentro de una organización en la gobernanza TI.

Palabras-clave: Gobernanza TI; identificación de necesidades; co-creación.

\section{The co-creation as a strategy to address IT governance in an organization}

\begin{abstract}
In common frameworks for Governance of Information Technology -ITa fundamental principle is related to identify and meet the needs of stakeholders inside and outside organizations. However, these frameworks do not document procedures to support the implementation. Co-creation is an interesting strategy to capture such requirements, allowing to generate a shared vision of the units of a company. This paper proposes a method based on co-creation to execute the needs identification phase in IT governance process. Five components for determining strategies, types of profiles of stakeholders, resources required for the implementation and motivation mechanisms are detailed. By employing the method, a significant increase in the flow of contributions, as well as creating innovative strategies to meet the needs within an organization in the IT governance were evident.
\end{abstract}

Keywords: IT Governance; needs identification; Co-Creation. 


\section{Introducción}

La información dentro de las organizaciones se configura como el recurso intangible más importante (Rojas, 2004), dado que permite apoyar la toma decisiones, bosquejar los horizontes del negocio y seleccionar las mejores alternativas para solucionar una necesidad particular. El apalancamiento de las Tecnologías de la Información y Comunicación -TI- es vital en el almacenamiento, gestión y análisis de este activo (A. Gómez \& Suárez, 2005), en orden de lograr los objetivos en pro del mejoramiento continuo, lo que redunda en la generación de valor (M. Gómez, Vicario, García, \& García, 2014; Huber, 1990).

Sin embargo, para poder generar un valor diferenciador en cada uno de los interesados al usar las TI dentro y fuera de una organización (evitando que éstas se conviertan paulatinamente en un problema), se requiere tener las metas estratégicas correctamente planificadas a través de estrategias de gobernanza claras, en las cuales todos los sectores de la empresa se suman, incluyendo la alta dirección y los ejecutivos (Van Grembergen \& De Haes, 2009), así como actores externos que hayan sido previamente seleccionados para participar en las dinámicas de la organización (Grossi \& Calvo-Manzano, 2011). La gobernanza en las TI se perfila como el medio para optimizar la creación de valor usando las TI. Esto por permitir minimización de los riesgos, maximización del uso de los recursos, y una visión holística desde la perspectiva organizacional.

Metodologías de gobernanza adoptadas globalmente tales como COBIT 5, ASA 8015 e IEC 38500 proponen diferentes principios de gobernabilidad TI, entre los que se cuenta como clave el de "satisfacer las necesidades de las partes interesadas" (Calder, 2008; De Haes, Van Grembergen, \& Debreceny, 2013; Too \& Weaver, 2014). Para una correcta identificación de expectativas e involucramiento de los interesados se propone incorporar el concepto de la co-creación para la realización de tareas colaborativas. Se trata de un paradigma que permite generar productos, servicios y/o procesos innovadores mediante la participación activa de todos los interesados, a partir del diálogo continuo, el acceso a la información, la gestión del riesgo y la transparencia (Prahalad \& Ramaswamy, 2004). Mecanismos adecuados de interacción entre los participantes en el contexto de la co-creación permitirán generar valor en las organizaciones (Gonzalez, Gonzalez, Echeverri, \& Urrego, 2013).

Surge el reto de formalizar y hacer operativa la construcción de entornos de co-creación y configurar este tipo de ejercicios (en modalidad presencial o virtual) a partir de una vista orientada por procesos, donde estén claramente definidos los aspectos críticos que facilitan la participación, el compromiso y la generación de valor, en orden de identificar las necesidades y posibles alternativas de solución en la gobernanza TI. Para tal efecto, el método propuesto está constituido por 5 subprocesos: selección de fases, selección de participantes y grupos, definición de técnicas de co-creación, selección de espacios, medios y tecnología y finalmente, definición de mecanismos de motivación y premiación. Se ofrece además un conjunto de guías, protocolos y listas de chequeo que facilitan la ejecución de los procesos. Se han hecho validaciones parciales en entornos reales para demostrar la efectividad del método propuesto.

Esta propuesta se constituye en una herramienta útil para las organizaciones que deseen incorporar de forma sistemática el concepto de co-creación de valor en la fase de identificación de necesidades en procesos de Gobernanza TI posibilitando el control, 
el seguimiento, la gestión y el perfeccionamiento de las actividades asociadas, con un consecuente mejoramiento de la creación de valor en estrategias de gobernanza TI.

El presente artículo se organiza como sigue: la sección 2 muestra el fundamento teórico en el cual se sustenta la propuesta. La sección 3 la metodología seguida para el estudio y presenta los hallazgos identificados en la revisión de la literatura, la evidencia empírica y la problemática identificada. La sección 4 detalla la solución propuesta. La sección 5 describe la validación de forma general. Finalmente se presentan las conclusiones, trabajo futuro, agradecimientos y referencias.

\section{Marco Teórico}

\subsection{Gobernanza de las Tecnologías de Información y Comunicación}

Se define como el sistema mediante el cual el uso presente y futuro de las TI es dirigido y controlado, el cual considera la evaluación y dirección de planes para su uso, en orden de apoyar los objetivos de la organización y monitorear su uso para ejecutar el plan, incluyendo estrategias y políticas transversales a la organización (Calder, 2008).

La alineación de las TI con la estrategia del negocio facilita la toma de decisiones, la asignación de roles proveedores de información relevante, el involucramiento de niveles directivos y operativos en procesos tan importantes como la mejora de procesos, entre otras ventajas (De Haes et al., 2013; Zhang \& Chulkov, 2011).

De acuerdo a diferentes metodologías para aplicar el concepto de gobernanza TI, uno de los primeros pasos es la identificación y satisfacción de necesidades de los involucrados (De Haes et al., 2013; Feltus, 2012; Zutshi, Creed, Wood, \& Couchman, 2009).

\subsection{Co-creación de valor}

El concepto hace alusión a la generación conjunta de valor a partir de la interacción entre agentes internos y externos a la organización. Esto se potencia mediante los principios de diálogo continuo, acceso a la información, riesgo/beneficio y transparencia (Prahalad \& Ramaswamy, 2004a). La co-creación se materializa a través de técnicas con esquema presencial como Knowledge SAFARI, Cognitive Edge Methods, por mencionar algunas (Samad, Wohlfart, \& Wolf, 2007). Un segundo tipo de co-creación es la mediada por TI (Gonzalez et al., 2013) tales como las redes sociales, los portales empresariales, los blogs, los mundos virtuales, teniendo en cuenta un conjunto de requerimientos funcionales y no funcionales (M. González, González, \& Urrego, 2012).

\subsection{Procesos de gobernanza TI mediados por la co-creación}

La identificación de necesidades y el análisis de oportunidades de la gobernanza TI puede desarrollarse empleando técnicas de co-creación. De esta manera las organizaciones se convierten en facilitadoras de entornos de experiencias donde los interesados expresan sus pensamientos, deseos, inconformidades y expectativas (Chen, Tsou, \& Ching, 2011). La intervención de las diferentes unidades dentro de la organización, así como el involucramiento de agentes externos durante las fases tempranas de cualquier proceso propician interacciones sistémicas y holísticas, tal como lo sugieren las metodologías para gobernanza TI (De Haes et al., 2013). 


\section{Metodología, revisión de referentes y hallazgos}

Con el propósito de proponer una nueva forma mediada por la co-creación para ejecutar la fase inicial del proceso de gobernanza TI en una organización, se empleó un enfoque abductivo (Thagard \& Shelley, 1997), donde se combinan propuestas disponibles en la literatura y experiencias de corte empírico (observación participativa). Con esto se logró extraer conocimiento de ambas fuentes, y extrapolar la evidencia recolectada de un dominio particular a otros dominios, obteniendo hallazgos preliminares (Aarikka-Stenroos \& Jaakkola, 2012; Dubois \& Gadde, 2002). La metodología es mostrada en la Figura 1.

La investigación se estructuró comenzando con la definición del contexto de interés, que para este caso hace referencia a la identificación de necesidades en la gobernanza TI. A partir de ello se procedió a ejecutar dos procesos de forma paralela: la revisión de literatura sobre propuestas para abordar la primera fase de un proceso de gobernanza TI; y la observación participativa en un conjunto de sesiones donde se capturaron necesidades en gobernanza TI. La información recopilada fue procesada y analizada mediante diagramas de afinidad (Shafer, Smith, \& Linder, 2005) para obtener un conjunto de recomendaciones. Lo siguiente fue proponer, a partir de los hallazgos, un método para identificar necesidades en gobernanza TI incorporando el concepto de co-creación. Por último se hicieron validaciones en ejercicios reales para medir la efectividad del método.

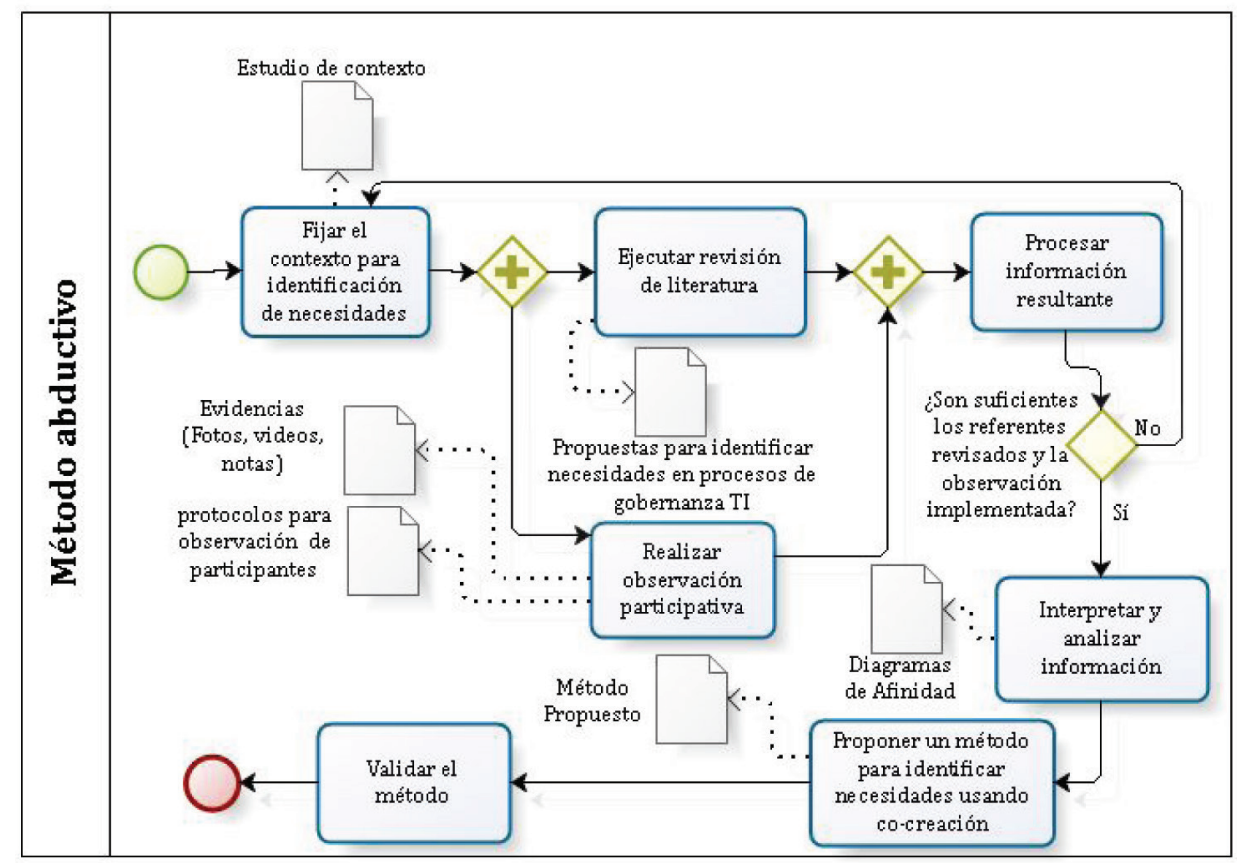

Figura 1 - Metodología empleada durante la investigación 
Durante la revisión de literatura se usó un método sistemático propuesto en la Universidad Politécnica de Valencia (MEDINA-LOPEZ, MARIN-GARCIA, \& ALFALLALUQUE, 2010), que parte de la identificación del campo de estudio y del período a analizar, posteriormente selecciona las fuentes de información. Luego realiza la búsqueda bajo parámetros previamente definidos resolviendo interrogantes asociados al qué, dónde y cómo. Seguidamente recomienda la ejecución de una prueba piloto para determinar la pertinencia de los descriptores de búsqueda definidos y ajustar en caso que sea necesario. Por último se documentan los resultados obtenidos en función de los elementos más relevantes a analizar.

Algunos descriptores de búsqueda usados se enuncian a continuación: governance IT, gorvernment ICT, co-creation, COBIT, stakeholders needs, governance principles. Las preguntas de investigación que orientaron la búsqueda fueron: ¿Cuáles son las estrategias usadas para identificar necesidades de las partes interesadas en procesos de gobernanza TI? ¿La co-creación es un medio adecuado para la identificación de dichas necesidades e incluso de la satisfacción de las mismas? De otro lado, la observación participativa fue el enfoque usado para la captura de información empírica y el trabajo de campo. En este caso, los investigadores prepararon en el año 2013 quince sesiones de co-creación presencial con diversos propósitos (siempre vinculados al tema de comercialización e innovación tecnológica). Allí se observaron los aportes en relación con la forma de hacer identificación de necesidades en procesos de gobernanza TI y detalles sobre la configuración de sesiones en orden a lograr mejores aportes para la identificación de necesidades. La información recopilada durante las sesiones fue grabada y transcrita para su posterior análisis. La observación se complementó con entrevistas estructuradas a expertos del dominio. El procesamiento de datos se hizo a partir de diagramas de afinidad (Shafer et al., 2005). El detalle no hace parte del alcance de este artículo.

\subsection{Hallazgos durante la revisión de literatura}

Con respecto a la gobernanza de las TI, se encontraron múltiples fuentes en las cuales se asevera que la identificación de necesidades debe ser ejecutada en las primeras fases de un proceso de gobernanza TI (Calder, 2008; De Haes et al., 2013; Feltus, 2012). En adición, se proponen diferentes roles dentro de la organización (interesados internos) y fuera de ella (interesados externos), así como un conjunto de preguntas orientadoras que permiten fijar las necesidades de los actores en cuanto a gobernanza TI, tales como: generación de valor usando TI, gestión del rendimiento usando TI, nuevas oportunidades de negocio mediante el uso de las TI, entre otros (ISACA, 2012). Sin embargo, no se presenta un marco de referencia para gestionar y configurar esta fase de forma adecuada (aportando detalles como espacios, técnicas a usar, herramientas, preguntas orientadoras, recomendaciones). La carencia de una metodología para la identificación de necesidades puede redundar en la incorrecta satisfacción de las mismas y, por ende, en una posible desalineación de las políticas de gobierno con los objetivos estratégicos del negocio.

\subsection{Hallazgos durante la observación participativa}

En los ejercicios se evidenciaron puntos de mejora en dos líneas:

- Aspectos clave para incorporar el concepto de co-creación durante la identificación de necesidades en gobernanza TI: 1) en organizaciones 
con un número considerable de unidades y empleados, las sesiones deben configurarse en sub-grupos de acuerdo a los perfiles, para evitar que algunas de las unidades no tengan la oportunidad de aportar; 2) para facilitar la co-creación de las políticas de gobernanza TI, un ejercicio de sensibilización introductorio (a manera de lúdica) permite poner en contexto a los participantes; 3) debe existir un rol asociado a la moderación de la sesión para evitar que la co-creación de políticas tome un rumbo incorrecto que no satisfaga las necesidades identificadas; 4) El uso de las preguntas orientadoras presentadas por marcos como COBIT 5 (ISACA, 2012) permiten orientar las sesiones de identificación de necesidades, sin embargo, se requiere una preparación adicional para conducirlas adecuadamente en una sesión de co-creación, evitando posibles improvisaciones.

- Expectativas de los participantes en procesos de gobernanza TI: Se evidenció que los interesados tienen las siguientes expectativas: 1) identificar la generación de valor usando $\mathrm{TI}$; 2) conocer el rendimiento del trabajo incorporando $\mathrm{TI}$; 3) observar las oportunidades de negocio asociadas a la incorporación de TI en la organización; 4) identificar los riesgos asociados a su utilización en el negocio; 5) proteger la información recolectada, procesada y analizada usando TI; 6) evitar que las TI se conviertan en obstáculos para los logros de la organización; 7) costear adecuadamente las inversiones en TI.

\subsection{Problemática identificada}

Al analizar los resultados obtenidos tanto en la revisión de literatura como en la observación participativa fue posible concluir que incorporar la gobernanza TI en las dinámicas de una organización es un proceso gradual (Carbonell, Rodríguez, \& Pujari, 2009; Magnusson, Matthing, \& Kristensson, 2003), y cualquiera sea la propuesta de gobernanza TI, no hay estrategias específicas para identificar y satisfacer necesidades, lo cual hace compleja la alineación con los objetivos del negocio. Esto hace que los resultados obtenidos varíen en función de variables no controladas, con las posteriores dificultades para replicar las experiencias exitosas o evitar malas prácticas debido a la falta de estandarización y lineamientos concretos (aunque COBIT 5 (ISACA, 2012) presenta preguntas orientadoras para la definición de necesidades no aborda detalles sobre cómo hacer operativa la identificación de necesidades). De otro lado, la interacción es posible siempre y cuando los participantes puedan concentrarse en un mismo lugar y tiempo, y esta condición difícilmente se cumple, máxime que hay gran variedad de organizaciones distribuidas en zonas geográficas alejadas entre ellas. Buscando resolver esta problemática se propone una nueva forma de identificar las necesidades de los interesados en la implementación de políticas y estrategias de gobernanza TI.

\section{Solución propuesta}

En esta sección se presenta un método para ejecutar la fase de identificación de necesidades de los interesados en un proceso de gobernanza TI. Se trata de incorporar el concepto de co-creación mediante un enfoque basado en procesos, donde será posible el control, seguimiento, gestión y perfeccionamiento de las actividades asociadas, con un consecuente mejoramiento de la competitividad. Esta aproximación ayuda a construir conjuntamente la política de gobierno dentro de una organización, alcanzando en 
muchos casos, resultados innovadores en el uso de las TI.

La notación empleada es el standard Business Process Model and Notation (BPMN). El método contiene 5 subprocesos (Figura 2) y uno de los principales productos que se obtiene es un documento maestro con todas las características y configuración de las sesiones de co-creación cuyo objetivo es orientar en la ejecución de la primera fase en un proceso de gobernanza TI.

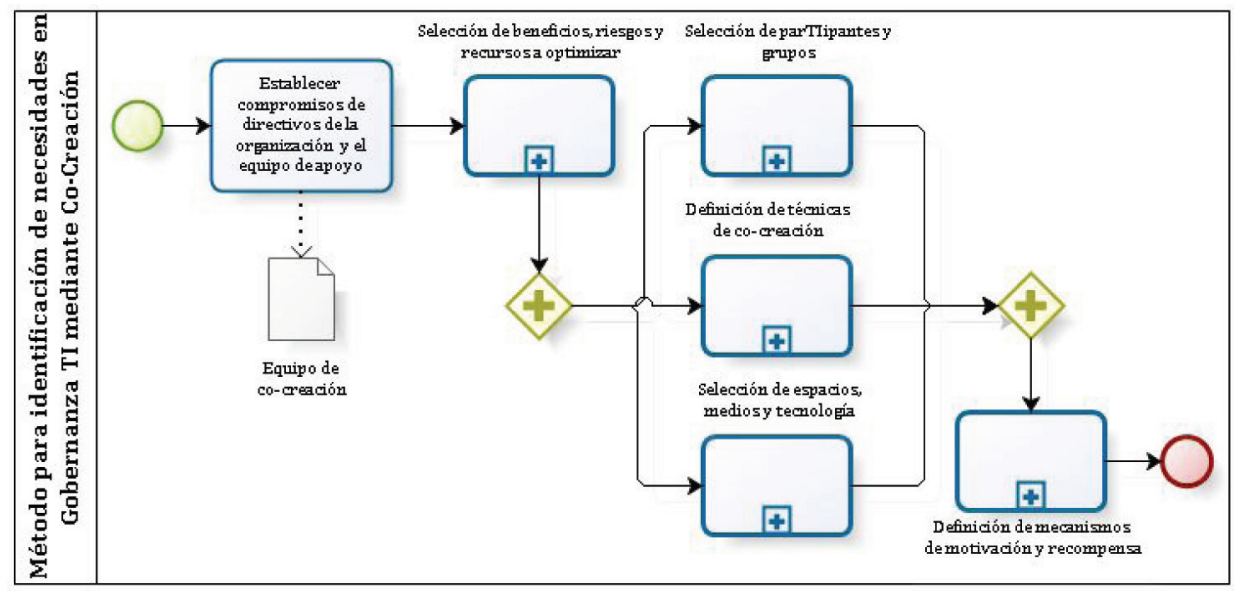

Figura 2 - Método para identificar necesidades en gobernanza TI a partir de co-creación

Antes de comenzar con el método y buscando ofrecer entornos adecuados de co-creación de experiencias y valor, se recomienda contar con la aprobación desde el nivel directivo lo cual garantiza el compromiso de los empleados. Lo siguiente es definir un grupo y su respectivo líder (en adelante denominado “coordinador de proceso de co-creación”) que acompañe todas las estrategias de generación de valor para la gobernanza TI. Este equipo debe ser seleccionado cuidadosamente y no al azar. Se trata de individuos con cultura de innovación (creativos, emprendedores, con pensamiento sistémico) que logren acercar a todos los interesados (Salomonson, Åberg, \& Allwood, 2012). Este grupo se encargará de definir los detalles para propiciar la interacción entre los interesados a partir del análisis hecho por el nivel directivo. De esto dependen otras decisiones que se tomen durante la identificación de necesidades para la gobernanza TI a partir de co-creación.

Lo siguiente es la "Selección de beneficios, riesgos y recursos a optimizar" (Figura 3). En este subproceso el nivel directivo debe establecer claramente los tópicos a co-crear (relacionados con los beneficios, recursos y riesgos esperados al incorporar la gobernanza TI) con los demás interesados dentro de la organización. Para ello contará con las capacidades del coordinador del proceso de co-creación, que a su vez puede apoyarse en guías brindadas por metodologías como COBIT (ISACA, 2012). Como producto de este subproceso se tendrá un documento maestro preliminar con un inventario inicial de necesidades considerando beneficios, riesgos y recursos. 


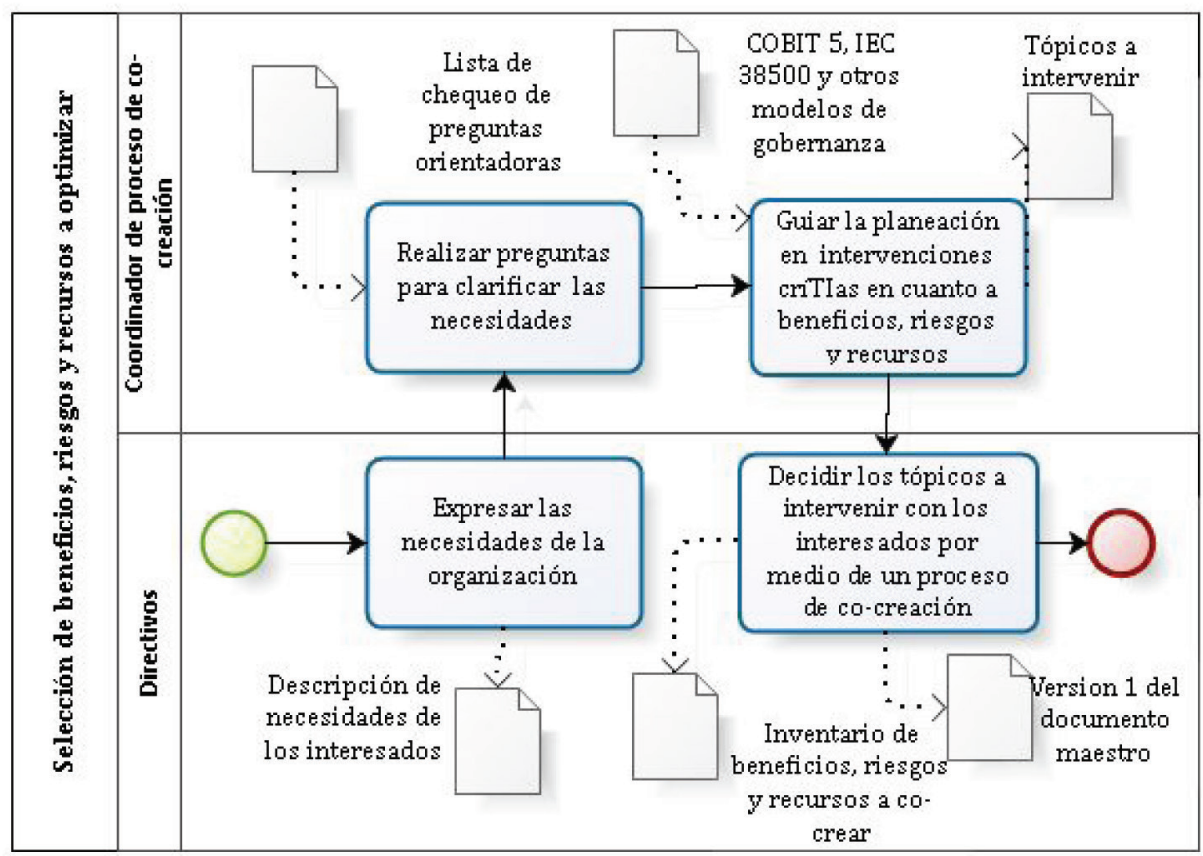

Figura 3 - Subproceso de selección de beneficios, riesgos y recursos a optimizar

Posteriormente se procede a la "Selección de participantes y grupos" (Figura 4). Es fundamental analizar muy bien las expectativas de la organización en cuanto a la Gobernanza TI, para definir el tipo de participantes en función a su conocimiento y aptitudes, cantidad conveniente de invitados y configuración del equipo de apoyo.

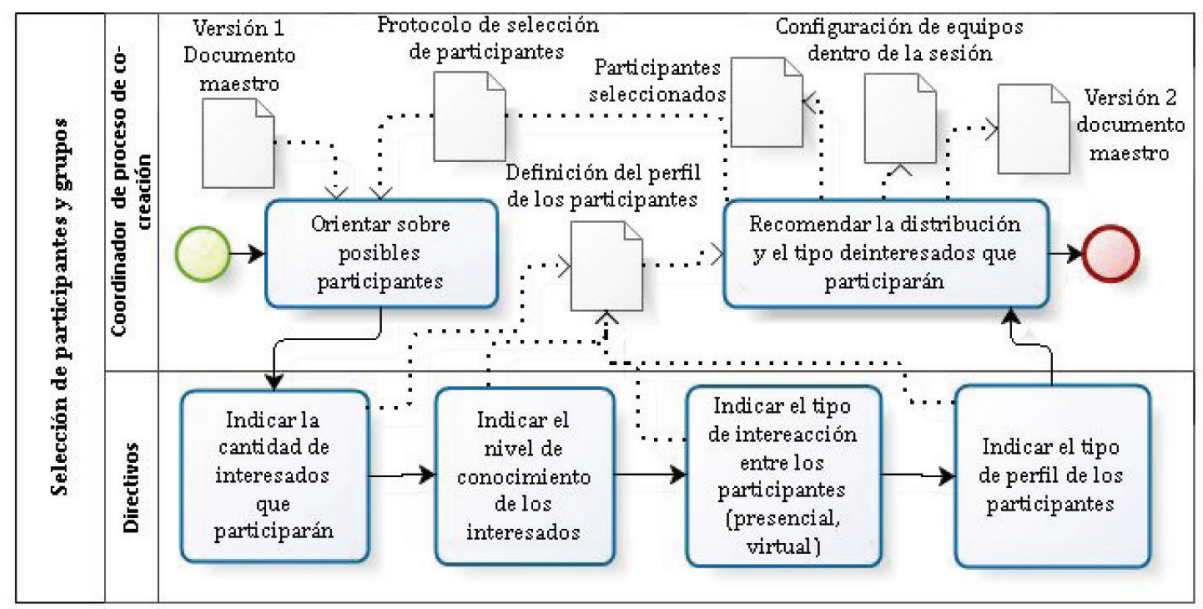

Figura 4 - Subproceso de selección de participantes y grupos 
Luego viene el subproceso "Definición de técnicas de co-creación" (Figura 5) cuyo objetivo es definir la técnica de co-creación apropiada para la sesión de generación de valor, en función de los parámetros establecidos previamente.

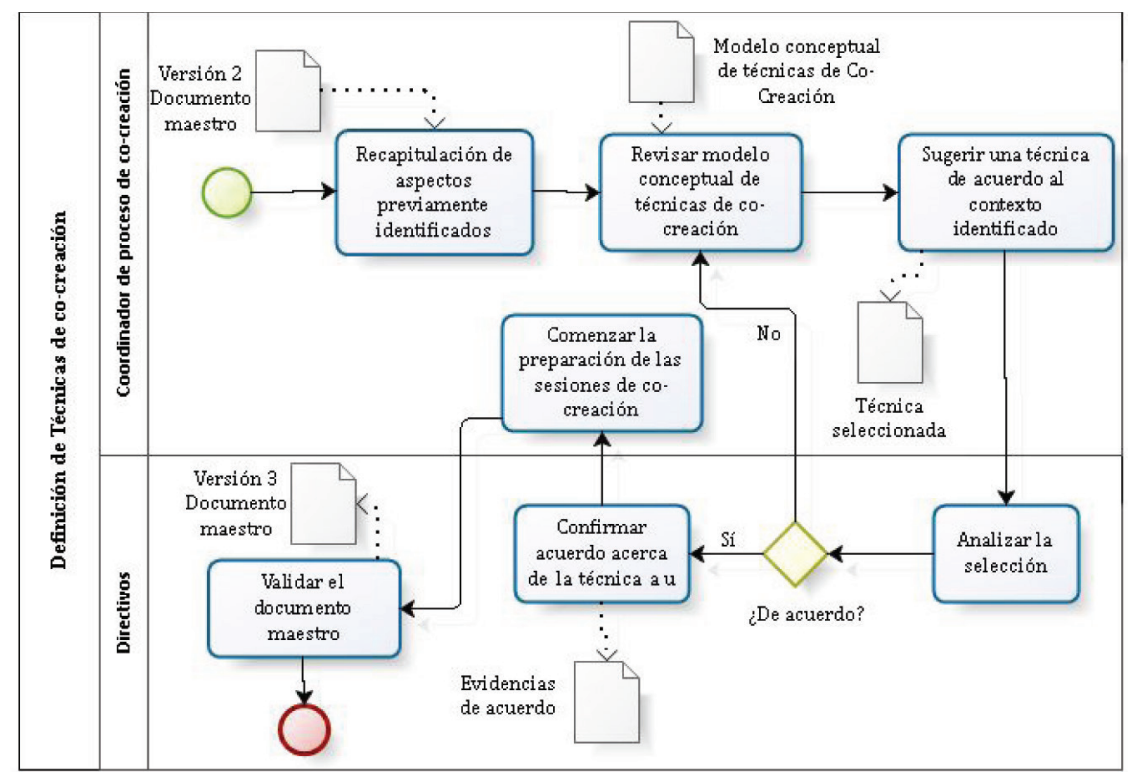

Figura 5 - Subproceso de definición de técnicas de co-creación

Buscando apoyar el subproceso anterior, durante esta investigación se generó un modelo conceptual con base a las recomendaciones presentadas en (Samad et al., 2007) con un conjunto de criterios y dimensiones que posibilitan una selección consciente y argumentada de la técnica de co-creación a usar (Figura 6). Este aporte se construyó a partir de la exploración y caracterización de aproximadamente 50 técnicas de co-creación, como se mostró previamente en (Gonzalez et al., 2013).

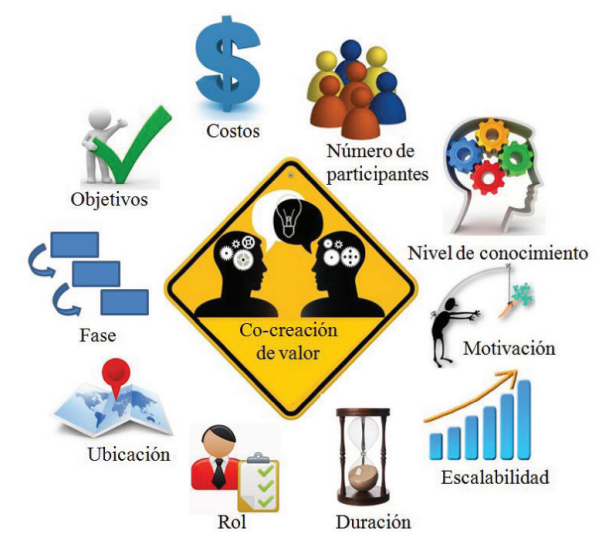

Figura 6 - Modelo conceptual con aspectos clave para seleccionar técnicas de co-creación 
Ante un escenario particular, se recomienda que el líder del equipo de co-creación haga la siguiente verificación buscando elegir la técnica adecuada: 1) Objetivos: hace referencia al problema que se desea solucionar mediante la co-creación, o el reto definido por los interesados. En general una técnica puede ser aplicada para (Samad et al., 2007): construir redes y conexiones entre participantes, generar conocimiento, facilitar el aprendizaje, generar estrategias, extraer conocimiento de los participantes, proponer mejores prácticas. En este trabajo particular se trabaja sobre la identificación de necesidades y generación de estrategias para satisfacerlas en procesos de gobernanza TI. 2) Número de participantes: cantidad recomendada de personas para aplicar la técnica de co-creación. 3) Nivel de conocimiento de los participantes: Se hace con respecto al conocimiento intrínseco de los participantes acerca de la organización y las necesidades de cada unidad individual. 4) Ubicación: lugar en el que se encuentran las personas que van a participar de la actividad y de qué forma se reúnen los participantes. Puede ser presencial, virtual o mixta. 5) Rol de los participantes: existen dos grandes grupos de interesados: el grupo 1 lo constituyen quienes coordinan la sesión de Co-creación, requiriéndose los siguientes roles: a) relatores (Toman apuntes de las ideas expresadas por los participantes, y se requiere uno por cada subgrupo); b) coordinador principal (orienta los diferentes momentos, ordena la actividad, fija normas, genera confianza entre los invitados), c) coordinadores de mesa (orientan la discusión en cada subgrupo para el ejercicio de co-creación, propicia el diálogo constructivo entres los participantes); d) personal de logística (se ocupa de garantizar disponibilidad de materiales preparativos de la sesión, protocolos y plantillas). El segundo grupo está conformado por los invitados a la sesión en calidad de participantes co-creadores. De acuerdo al objetivo del ejercicio es posible invitar actores internos y externos a la organización, ayudando a alinear las necesidades y generación de valor en la Gobernanza TI. 6) Duración: Tiempo que se emplea para aplicar la técnica de Co-creación. Puede ejecutarse en menos de 1 hora, entre $1-4$ horas, entre $1-3$ días, de 1- 6 semanas, o incluso en meses. 7) Costo: la inversión en la ejecución de la técnica puede ser bajo, medio, alto. 8) Escalabilidad: Aunque esta característica puede estar incluida en el número de participantes, es importante tener una variable separada para identificar qué tan escalable es una técnica y qué tanto se adapta al cambio de participantes.

El método se complementa con el subproceso "Selección de espacios, medios y tecnología" (Figura 7) buscando mantener un diálogo continuo con los interesados, fomentando la transparencia, el acceso y la visualización de experiencias. Hay variaciones sustanciales de acuerdo al estilo de la interacción. Mientras que una sesión de co-creación presencial es apoyada con recursos físicos como cartulinas, post-it, marcadores, lapiceros, un ejercicio mediado por TI se sirve de redes sociales, blogs, portales empresariales, sistemas de videoconferencia, chats, aplicaciones móviles, etc. Los dos tipos de interacción se tienen en cuenta en el proceso sugerido.

Como apoyo a este subproceso se emprendió la exploración detallada de un conjunto de portales empresariales de co-creación, redes sociales y aplicaciones móviles buscando ofrecer opciones de interacción distribuida y mediada por TI. El detalle de las listas de chequeo y catálogos enunciadas en la Figura 7 fue previamente divulgado en (Gonzalez et al., 2013). 


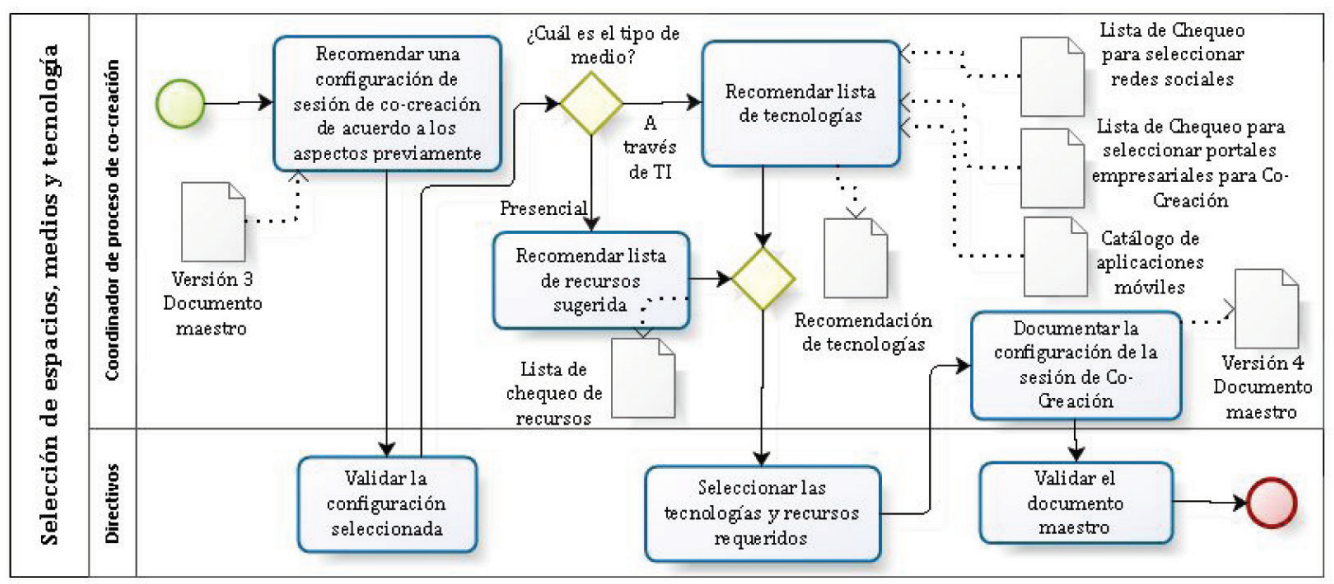

Figura 7 - Subproceso de selección de espacios, medios y tecnología

El último subproceso es la "Definición de mecanismos de motivación y premiación", buscando lograr un clima de confianza y expectativa entre los participantes y de esta manera potenciar la generación de aportes. Se trata de una etapa crítica para el éxito de una estrategia de co-creación cualquiera sea su propósito. En la Figura 8 se muestra la dinámica de ejecución sugerida.

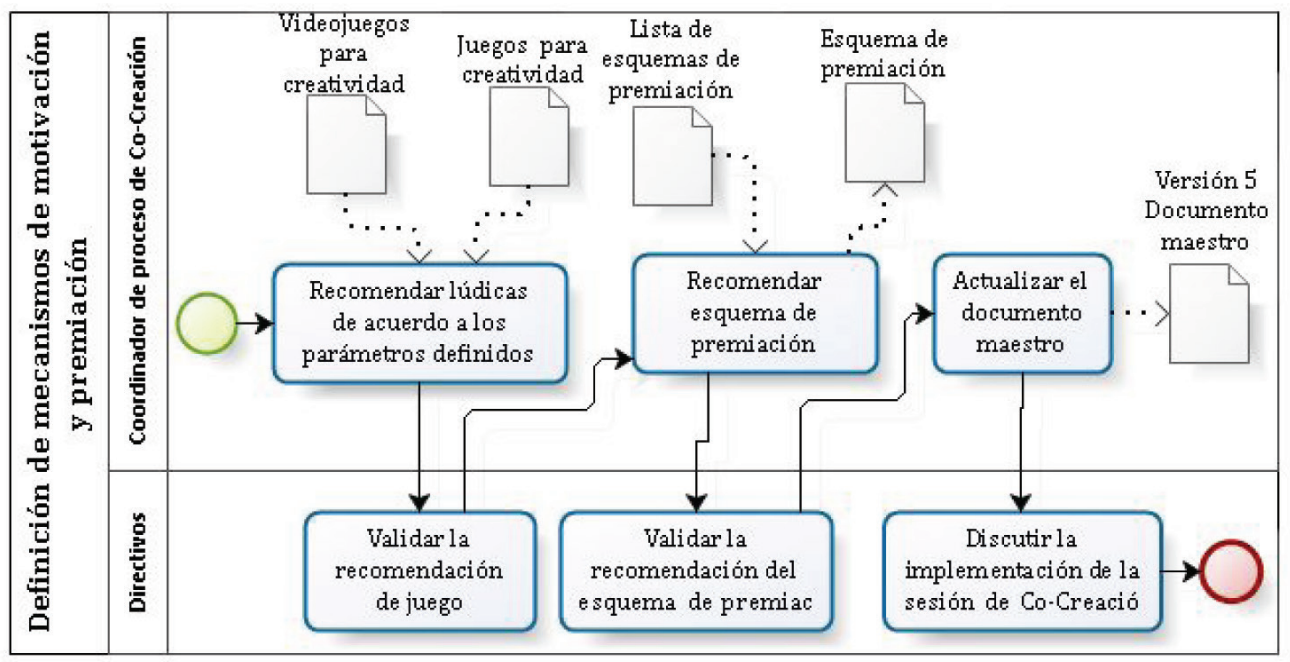

Figura 8 - Subproceso de definición de mecanismos de motivación y premiación 
En cuanto al aspecto motivacional se sugiere incorporar el componente lúdico para ambos tipos de interacción (presencial y mediada por TI). La sesión de cocreación debería iniciar con un juego que incentive el pensamiento lateral y el trabajo colaborativo. Esto será el motor para la generación de aportes durante la resolución de problemas abiertos (como el escenario de identificación y satisfacción de necesidades en Gobernanza TI) en contraposición al pensamiento analítico-racional, adecuado para resolver problemas con soluciones preestablecidas (De Bono, 1990). Algunas estrategias ya identificadas para estimular el pensamiento lateral y el trabajo colaborativo son: acertijos, ilusiones ópticas, imágenes y a partir de ellas hacer una descripción, frases inductoras, juegos, cuestionarios con preguntas fuera del contexto de aplicación. Para orientar a los responsables del proceso de co-creación también se cuenta con un listado de posibles estrategias y juegos pero por temas de espacio no serán presentados en la presente contribución.

Con respecto a la premiación, durante el estudio de portales de co-creación enunciado anteriormente (Gonzalez et al., 2013) se logró detectar que las empresas premian a los participantes mediante (Antikainen, 2011): remuneración monetaria (esquema más usado), mejora en su reputación o esquemas de puntos, propuesta de patentes conjuntas, desarrollo y maduración de la idea planteada con patrocinadores, posibilidades laborales. Una vez se definen estos detalles se debe hacer una actualización del documento maestro, para efectos de ajustar tiempos y recursos (logísticos, humanos). Con este subproceso se da por terminado el método propuesto para la identificación de necesidades en procesos de gobernanza TI, mediante enfoque de co-creación de valor.

\section{Validación del método}

La validación de la propuesta se hizo mediante 10 casos de estudio donde se configuraron sesiones para identificar necesidades en procesos de gobernanza TI de organizaciones puntuales, atendiendo las indicaciones ofrecidas por el método. Fue posible evidenciar un aumento significativo en el flujo de aportes, además de mejorar la percepción de los participantes al detectar configuraciones ordenadas y sistemáticas para estos ejercicios donde se busca generar entornos de confianza y propiciar una construcción conjunta de necesidades, entre empleados e interesados externos en procesos de gobernanza TI. Uno de los escenarios de validación permitió construir por completo el "Modelo de comercialización tecnológica para la región antioqueña”, proyecto de gran envergadura donde participaron actores de diversos tipos (universidades, empresas, centros de desarrollo tecnológico, sector gobierno). En la Figura 9 se presentan algunas evidencias de las sesiones ejecutadas siguiendo el método.

\section{Conclusiones}

En esta investigación se propuso un método para identificar las necesidades de los interesados en procesos de Gobernanza TI mediante el uso de sesiones de co-creación bajo modalidad presencial o virtual. Para ello se estructuró un proceso principal y cinco subprocesos. En trabajos previos (L. González, 2012; Gonzalez et al., 2013) se ofrece además un conjunto de protocolos, listas de chequeo y plantillas para facilitar la toma 

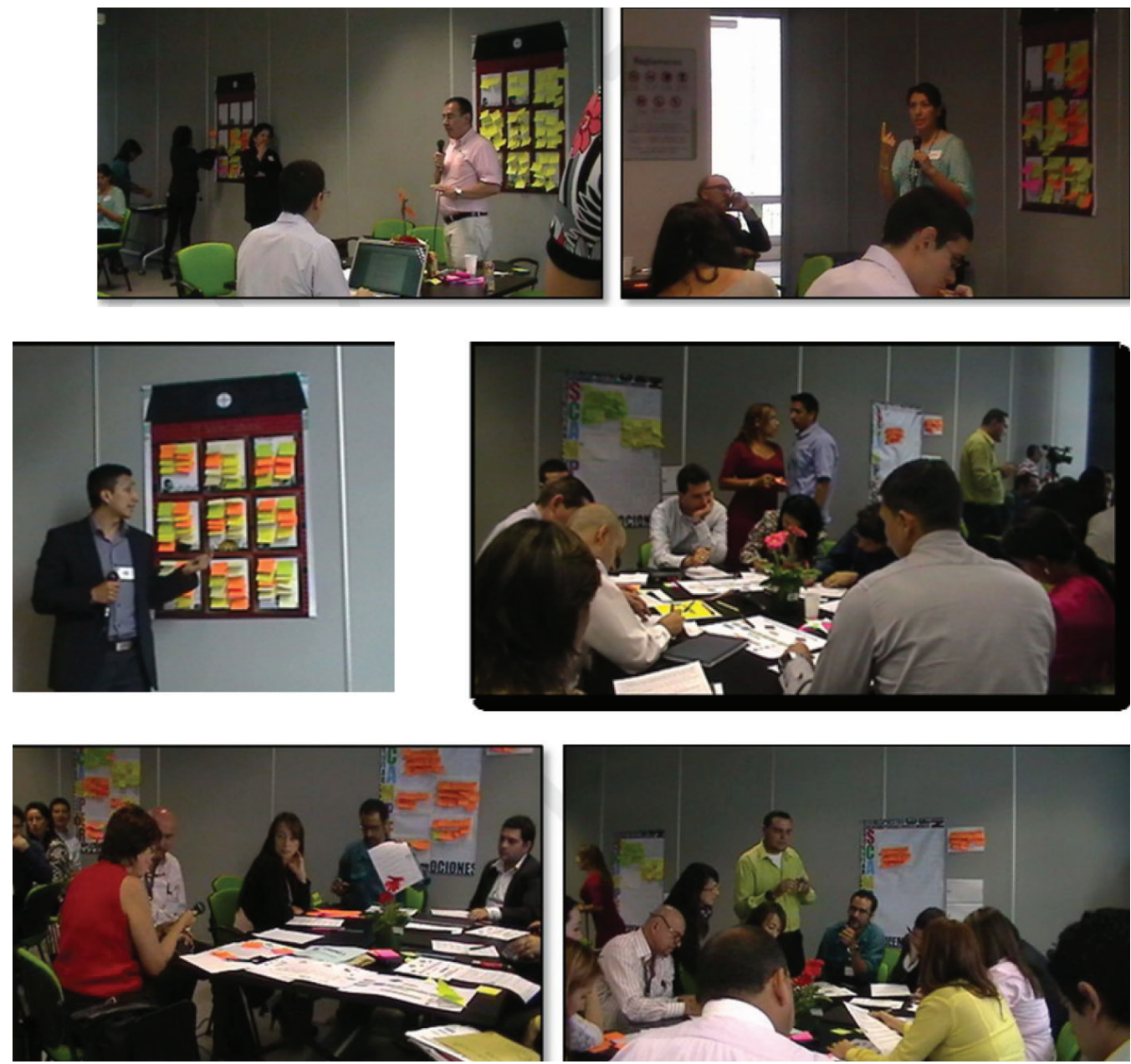

Figura 9 - Algunos escenarios de validación del método propuesto

de decisiones (en cuanto a herramientas, medios y tecnologías). Fue posible evidenciar que para configurar adecuadamente un ejercicio de co-creación es necesario en primera instancia construir un inventario de beneficios, riesgos y recursos con el equipo directivo de una organización. Posteriormente se debe seleccionar cuidadosamente quiénes participarán en la iniciativa y cómo se conformarán los grupos de trabajo. Con estos parámetros definidos, es necesario escoger las técnicas de co-creación a aplicar. Se requiere también definir los espacios, medios, recursos y tecnología que soportarán la sesión. Existen innumerables plataformas de apoyo a la co-creación, entre ellas, las redes sociales, los portales empresariales de co-creación y las aplicaciones móviles cuyo propósito es facilitar la generación de ideas y el intercambio colaborativo. Por último, pero no menos importante, es fundamental definir los mecanismos de motivación y premiación. 
El método propuesto ayuda y mejora el proceso de identificación y satisfacción de necesidades en las fases previas de la gobernanza TI, en tanto ofrece mecanismos claros y organizados para facilitar la interacción entre las partes interesadas, ayuda a generar debate acerca de los objetivos estratégicos de la organización en cuanto a Tecnologías de Información y Comunicación, y en adición, permite generar innovaciones conjuntas en los procesos apoyados por TI. Es importante no perder de vista que el éxito de un proceso de co-creación depende, fundamentalmente, de la adecuada gestión del proceso (Samad et al., 2007). Por lo tanto, y a diferencia de lo que muchas empresas suponen, no se trata de ejercicios sin planear. Se deben concentrar esfuerzos importantes para garantizar una interacción sin limitantes de tiempo, lugar o medio de acceso (Antikainen, 2011). De igual manera, la Gobernanza de TI requiere metodologías estandarizadas para generar impacto.

\section{Trabajo Futuro}

Se planea estructurar otras sesiones de co-creación para detectar necesidades en Gobernanza TI empleando el método propuesto. Es necesario contar con un diseño experimental riguroso buscando validar su efectividad ya que en los ejercicios realizados no se determinaron variables a medir por lo tanto no es posible establecer comparaciones. Se desea determinar su aplicabilidad en diferentes nichos. También se emprenderá la construcción de una plataforma tecnológica para ofrecer este tipo de servicio soportado por las TI.

\section{Agradecimientos}

Al programa Enlaza Mundos de la Alcaldía de Medellín- modalidad pasantías.

\section{Referencias}

Aarikka-Stenroos, L., \& Jaakkola, E. (2012). Value co-creation in knowledge intensive business services: A dyadic perspective on the joint problem solving process. Industrial Marketing Management, 41(1), 15-26. doi: http://dx.doi.org/10.1016/j. indmarman.2011.11.008

Antikainen, M. (2011). Facilitating customer involvement in collaborative online innovation communities. (PhD), Tampere University, Tampere.

Calder, A. (2008). ISO/IEC 38500: the IT governance standard: IT Governance Ltd.

Carbonell, P., Rodríguez, A., \& Pujari, D. (2009). Customer involvement in new service development: An examination of antecedents and outcomes. Journal of Product Innovation Management, 29(5), 536-550. doi: http://dx.doi.org/10.1111/j.15405885.2009.00679.x

Chen, J.-S., Tsou, H.-T., \& Ching, R. K. H. (2011). Co-production and its effects on service innovation. Industrial Marketing Management, 4O(8), 1331-1346. doi: http://dx.doi.org/10.1016/j.indmarman.2011.03.001 
De Bono, E. (1990). Lateral Thinking: Creativity Step by Step. New York: Harper Colophon.

De Haes, S., Van Grembergen, W., \& Debreceny, R. S. (2013). COBIT 5 and enterprise governance of information technology: Building blocks and research opportunities. Journal of Information Systems, 27(1), 307-324. doi: http://dx.doi.org/10.2308/isys50422

Dubois, A., \& Gadde, L.-E. (2002). Systematic combining: an abductive approach to case research. Journal of Business Research, 55(7), 553-560. doi: http://dx.doi. org/10.1016/S0148-2963(00)00195-8

Feltus, C. (2012). Introducing ISO/IEC 38500: Corporate Governance in ICT. ITSMF Jaarcongres 2008, 27-28.

Gómez, A., \& Suárez, C. (2005). Sistemas de información: herramientas prácticas para la gestión empresarial. Madrid: Ra-Ma Editorial.

Gómez, M., Vicario, B., García, C., \& García, A. (2014). Percepciones de los empresarios de Pymes rurales sobre la integración de las TIC. RISTI - Revista Ibérica de Sistemas e Tecnologias de Informação, (E2), 71-84. doi: http://dx.doi.org/10.17013/ risti.e2.71-84.

González, L. (2012). PROPOSAL OF A MODEL OF INNOVATION PROCESS WITH FOCUS IN CO-CREATION. In G. Gonzalez-Calderón \& C. Zapata (Eds.), Innovative ways of knowledge representation and management (Vol. , pp. 222). Medellín: Sello Editorial UdeM.

Gonzalez, L., Gonzalez, M., Echeverri, J., \& Urrego, G. (2013, 19-22 June 2013). Method of interaction in open innovation processes incorporating ubiquitous environments and web 2.o social networks: A baseline architecture. Paper presented at the Information Systems and Technologies (CISTI), 2013 8th Iberian Conference on.

González, M., González, L., \& Urrego, G. (2012). Non-Functional Requirements of UbiPlatInno: Ubiquitous Platform which supports Co-Creation. Software Engineering: Methods, Modeling, And Teaching, 61.

Grossi, L., \& Calvo-Manzano, J. A. (2011). Análisis de decisiones en la selección de proveedores de tecnologías de la información: una revisión sistemática. RISTI Revista Ibérica de Sistemas e Tecnologias de Informação,(8), 67-79.

Huber, G. P. (1990). A theory of the effects of advanced information technologies on organizational design, intelligence, and decision making. Academy of management review, 15(1), 47-71.

ISACA. (2012). Cobit 5: Un Marco de Negocio para el Gobierno y la Gestión de las TI de la Empresa.

Magnusson, P., Matthing, J., \& Kristensson, P. (2003). Managing user involvement in service innovation. Journal of Service Research, 6(2), 111-124. doi: http://dx.doi. org/10.1177/1094670503257028 
MEDINA-LOPEZ, C., MARIN-GARCIA, J., \& ALFALLA-LUQUE, R. (2010). A methodological proposal for the systematic literature review. WPOM-Working Papers on Operations Management, 1(2), 13-30.

Prahalad, C., \& Ramaswamy, V. (2004). Co-creation experiences: The next practice in value creation. Journal of Interactive Marketing, 18(3), 5-14. doi: http://dx.doi.org/ citeulike-article-id:894137

Prahalad, C., \& Ramaswamy, V. (2004a). Co-Creation Experiences: The Next Practice in Value Creation. Journal of Interactive Marketing, 18(3).

Rojas, Y. (2004). Organización de la información: un factor determinante en la gestión empresarial. ACIMED, 12(2), 1-1.

Salomonson, N., Åberg, A., \& Allwood, J. (2012). Communicative skills that support value creation: A study of $\mathrm{B} 2 \mathrm{~B}$ interactions between customers and customer service representatives. Industrial Marketing Management, 41(1), 145-155. doi: http://dx.doi.org/10.1016/j.indmarman.2011.11.021

Samad, A., Wohlfart, L., \& Wolf, P. (2007). Hands-On Knowledge Co-Creation and Sharing: Practical Methods and Tecniques. Stuttgart: KnowledgeBoard.

Shafer, S. M., Smith, H. J., \& Linder, J. C. (2005). The power of business models. Business Horizons, 48(3), 199-207.

Thagard, P., \& Shelley, C. (1997). Abductive reasoning: Logic, visual thinking, and coherence Logic and scientific methods (pp. 413-427): Springer.

Too, E. G., \& Weaver, P. (2014). The management of project management: a conceptual framework for project governance. International Journal of Project Management, 32(8), 1382-1394. doi: http://dx.doi.org/10.1016/j.ijproman.2013.07.006

Van Grembergen, W., \& De Haes, S. (2009). Enterprise governance of information technology: achieving strategic alignment and value: Springer Science \& Business Media.

Zhang, Y., \& Chulkov, N. (2011). Gestión de la tecnología de la información y las comunicaciones en las organizaciones del sistema de las Naciones Unidas.

Zutshi, A., Creed, A., Wood, G., \& Couchman, P. (2009). Relevance and necessity of standards for ICT governance. Paper presented at the ANZAM 2009: Integration through innovation: program and abstracts of the ANZAM Operations, supply chain and servics management symposium 2009 8th and 10th June 2009 Adelaide, South Australia. 